The location of the curve on the $\theta$ axis can be shifted to larger angles by increasing $V_{2}$ and $R$ (thus maintaining the well-known $V R$ ambiguity in the optical model) and to smaller angles by increasing $V_{1}$ and $|\eta|$, the energy difference between entrance and exit channels, which is determined experimentally and not treated as a parameter. The effect of varying $V_{2}$ is much larger than that of varying $V_{1}$, since $V_{2}$ determines two opticalmodel wave functions, $V_{1}$ only determines one. It was found that a large difference between $V_{2}$ and $V_{1}$ was necessary to locate the curves proper1y. The values quoted are not unique.

The over-all width is determined almost exclusively by $R_{b}$. Increasing $R_{b}$ decreases the overall width and increases the magnitude of the cross section at the center of the curve. It is found that when the best value of $R_{b}$ is used in each state, the relative magnitudes are automatically fitted well.

The effects of increasing $W_{1}, W_{2}$, and $a$ are small. Increasing $W_{1}$ and $W_{2}$ decreases the magnitude of both curves slightly. In fitting the $p$ state curve, $V_{2}$ and $V_{1}$ have opposite effects on the ratio of peak heights. Increasing $V_{2}$ increases the ratio. Increasing both $V_{1}$ and $V_{2}$ reduces the depth of the minimum by a very small amount.

The physical conclusions which we tentatively draw from this calculation are rather significant. For finite potentials there cannot be significant differences between single-particle wave functions whose principal quantum number, angular momentum, binding energy, and rms radius are given. Hence it seems that a distorted-wave analysis of $(p, 2 p)$ experiments determines the single-particle wave functions very well.

The rms radius of the charge distribution in $\mathrm{C}^{12}$ given by our empirical values of $R_{b}$ is $2.5 \mathrm{~F}$. The experimental value obtained from electron scattering is $2.4 \mathrm{~F}$. The $\mathrm{rms}$ radius for $s$-state protons is $1.7 \mathrm{~F}$, which is the experimental value for the $\alpha$ particle. Whether this is true for $s$ states in other light nuclei is, at present, being investigated by a systematic study of the available data. Finer points concerning curve fitting are also being investigated.

We would like to thank Dr. M. A. Melkanoff, Dr. J. S. Nodvik, Dr. D. S. Saxon, and Dr. D. G. Cantor for the use of their optical-model code SCAT 4 which was used to calculate our opticalmodel wave functions, and Dr. C. A. Hurst and $\mathrm{Mr}$. K. A. Amos for valuable discussions.

\footnotetext{
*Work supported in part by the Australian Institute for Nuclear Science and Engineering and a Colombo Plan scholarship.

${ }^{\dagger}$ Permanent address: University of Malaya, Kuala Lumpur, Malaya.

†Permanent address: University of California, Davis, California.

${ }^{1}$ A. J. Kromminga and I. E. McCarthy, Phys. Rev. Letters $\underline{4}, 288(1960)$.

${ }^{2}$ K. F. Riley, H. G. Pugh, and T. J. Gooding, Nucl. Phys. 18, 65 (1960); T. Berggren and G. Jacob, Phys. Letters 1 , 258 (1962); K. L. Lim and I. E. McCarthy, Proceedings of the International Symposium on Direct Interactions and Nuclear Reaction Mechanisms, Padua, 1962 (Gordon and Breach, New York, 1963). Further references are given in these papers.

${ }^{3}$ J. P. Garron, J. C. Jacmart, M. Riou, C. Ruhla, J. Teillac, and K. Strauch, Nucl. Phys. 37, 126 (1962).
}

\title{
UNITARY SYMMETRY AND LEPTONIC DECAYS
}

\author{
Nicola Cabibbo \\ CERN, Geneva, Switzerland \\ (Received 29 April 1963)
}

We present here an analysis of leptonic decays based on the unitary symmetry for strong interactions, in the version known as "eightfold way,", and the $V-A$ theory for weak interactions. ${ }^{2,3}$ Our basic assumptions on $J_{\mu}$, the weak current of strong interacting particles, are as follows:

(1) $J \mu$ transforms according to the eightfold representation of $\mathrm{SU}_{3}$. This means that we neglect currents with $\Delta S=-\Delta Q$, or $\Delta I=3 / 2$, which should belong to other representations. This limits the scope of the analysis, and we are not able to treat the complex of $K^{0}$ leptonic decays, or $\Sigma^{+} \rightarrow n+e^{+}+\nu$ in which $\Delta S=-\Delta \boldsymbol{Q}$ currents play a role. For the other processes we make the hypothesis that the main contributions come from that part of $J_{\mu}$ which is in the eightfold representation.

(2) The vector part of $J_{\mu}$ is in the same octet as the electromagnetic current. The vector contribution can then be deduced from the electromagnetic properties of strong interacting particles. For $\Delta S=0$, this assumption is equivalent to vector- 
current conservation. ${ }^{2}$

Together with the octet of vector currents, $j_{\mu}$, we assume an octet of axial currents, $g_{\mu}$. In each of these octets we have a current with $\Delta S$ $=0, \Delta Q=1, j_{\mu}{ }^{(0)}$, and $g_{\mu}{ }^{(0)}$, and a current with $\Delta S=\Delta Q=1, j_{\mu}^{(\mathbf{1})}$, and $g_{\mu}^{(\mathbf{1})}$. Their isospin selection rules are, respectively, $\Delta I=1$ and $\Delta I=1 / 2$.

From our first assumption we then get

$$
J_{\mu}=a\left(j_{\mu}^{(0)}+g_{\mu}^{(0)}\right)+b\left(j_{\mu}^{(1)}+g_{\mu}^{(1)}\right) \text {. }
$$

A restriction $a=b=1$ would not ensure universality in the usual sense (equal coupling for all currents), because if $J_{\mu}$ [as given in Eq. (1)] is coupled, we can build a current, $b\left(j_{\mu}{ }^{(0)}+g_{\mu}{ }^{(0)}\right)$ $-a\left(j_{\mu}^{(1)}+g_{\mu}^{(1)}\right)$, which is not coupled. We want, however, to keep a weaker form of universality, by requiring the following:

(3) $J \mu$ has "unit length," i.e., $a^{2}+b^{2}=1$.

We then rewrite $J_{\mu}$ as

$$
J_{\mu}=\cos \theta\left(j_{\mu}^{(0)}+g_{\mu}{ }^{(0)}\right)+\sin \theta\left(j_{\mu}{ }^{(1)}+g_{\mu}{ }^{(1)}\right),
$$

where $\tan \theta=b / a$. Since $J_{\mu}$, as well as the baryons and the pseudoscalar mesons, belongs to the octet representation of $\mathrm{SU}_{3}$, we have relations (in which $\theta$ enters as a parameter) between processes with $\Delta S=0$ and processes with $\Delta S=1$.

To determine $\theta$, let us compare the rates for $K^{+} \rightarrow \mu^{+}+\nu$ and $\pi^{+} \rightarrow \mu^{+}+\nu$; we find

$$
\begin{aligned}
& \Gamma\left(K^{+} \rightarrow \mu \nu\right) / \Gamma\left(\pi^{+}-\mu \nu\right) \\
& \quad=\tan ^{2} \theta M_{K}\left(1-M_{\mu}{ }^{2} / M_{K}{ }^{2}\right)^{2} / M_{\pi}\left(1-M_{\mu}{ }^{2} / M_{\pi}{ }^{2}\right)^{2}
\end{aligned}
$$

From the experimental data, we then $\operatorname{get}^{5}, 6$

$$
\theta=0.257 \text {. }
$$

For an independent determination of $\theta$, let us consider $K^{+} \rightarrow \pi^{0}+e^{+}+\nu$. The matrix element for this process can be connected to that for $\pi^{+} \rightarrow \pi^{0}$ $+e^{+}+\nu$, known from the conserved vector-current hypothesis (2nd assumption). From the rate ${ }^{6}$ for $K^{+} \rightarrow \pi^{0}+e^{+}+\nu$, we get

$$
\theta=0.26 \text {. }
$$

The two determinations coincide within experimental errors; in the following we use $\theta=0.26$.

We go now to the leptonic decays of the baryons, of the type $A-B+e+\nu$. The matrix element of any member of an octet of currents among two baryon states (also members of octets) can be expressed in terms of two reduced matrix elements ${ }^{7}$

$$
\left\langle A\left|j{ }_{\mu}^{(i)}+g_{\mu}^{(i)}\right| B\right\rangle=i f_{A B i}{ }^{O}{ }_{\mu}+d_{A B i}{ }^{E}{ }_{\mu} ;
$$

the $f$ 's and $d$ 's are coefficients defined in GellMann's paper. ${ }^{1,7}$ It is sufficient to consider only allowed contributions and write

$$
O_{\mu}, E_{\mu}=F^{O, E_{\mu}} \gamma^{O, E_{\gamma}} \gamma_{\mu}{ }_{5} .
$$

From the connection with the electromagnetic current we get the vector coefficients: $F^{O}=1$, $F^{E}=0$; from neutron decay we get

$$
H^{O}+H^{E}=1.25 \text {. }
$$

We remain with one parameter which can be determined from the rate for $\Sigma^{-} \rightarrow \Lambda+e^{-}+\bar{\nu}$. The relevant matrix element for this is

$$
\begin{aligned}
\cos \theta\left\langle\Sigma^{-}\left|j_{\mu}{ }^{(0)}+g_{\mu}{ }^{(0)}\right| \Lambda\right\rangle & \\
& =\cos \theta\left(\frac{2}{3}\right)^{1 / 2} E_{\mu}=\left(\frac{2}{3}\right)^{1 / 2} \cos \theta H^{E} \gamma_{\mu} \gamma_{5} .
\end{aligned}
$$

Taking the branching ratio for this mode to be $0.9 \times 10^{-4}$, $^{8}$ we get

$$
H^{E}= \pm 0.95 \text {. }
$$

The negative solution can be discarded because it produces a large branching ratio for $\Sigma^{-} \rightarrow n$ $+e^{-}+\bar{\nu}$, of the order of $1 \%$. The positive solution $\left(H^{E}=0.95, H^{O}=0.30\right)$ is good, because it produces a cancellation of the axial contribution to this process. This explains the experimental result that this mode is more depressed than the $\Lambda \rightarrow p+e^{-}+\bar{\nu}$ in respect to the predictions of Feynman and Gell-Mann. ${ }^{2}$ In Table I we give a summary of our predictions for the electron modes with $\Delta S=1$. The branching ratios for $\Lambda \rightarrow p+e^{-}+\bar{\nu}$ and $\Sigma^{-} \rightarrow n+e^{-}+\bar{\nu}$ are in good agreement with experimental data. ${ }^{9}$

As a final remark, the vector-coupling constant for $\beta$ decay is not $G$, but $G \cos \theta$. This gives a correction of $6.6 \%$ to the $f t$ value of Fermi transitions, in the right direction to eliminate the discrepancy between $\mathrm{O}^{14}$ and muon lifetimes.

Table I. Predictions for the leptonic decays of hyperons.

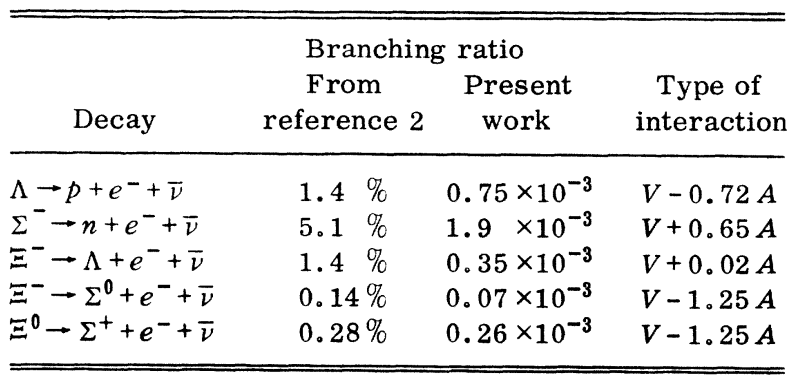


The correction is, however, too large, leaving about $2 \%$ to be explained. ${ }^{10}$

\footnotetext{
${ }^{1} \mathrm{M}$. Gell-Mann, California Institute of Technology Report CTSL-20, 1961 (unpublished); Y. Ne'eman, Nucl. Phys。26, 222 (1961).

${ }^{2}$ R. P. Feynman and M. Gell-Mann, Phys。Rev。 109,193 (1958).

${ }^{3}$ R. E. Marshak and E. C. G. Sudarshan, Proceedings of the Padua-Venice Conference on Mesons and Recently Discovered Particles, September, 1957 (Società Italiana di Fisica, Padua-Venice, 1958); Phys. Rev。109, 1860 (1958).

${ }^{4}$ Similar considerations are forwarded in M. GellMann and M. Lévy, Nuovo Cimento 16, 705 (1958).

${ }^{5}$ The lifetimes from W. H. Barkas and A. H. Rosenfeld, Proceedings of the Tenth Annual International Rochester Conference on High-Energy Physics, 1960 (Interscience Publishers, Inc., New York, 1960), p. 878. The branching ratio for $K^{+} \rightarrow \mu^{+}+\nu$ is taken as $57.4 \%$. W. Becker, M. Goldberg, E. Hart, J. Leitner, and S. Lichtman (to be published).

${ }^{6}$ B. P. Roe, D. Sinclair, J. L. Brown, D. A. Glaser, J. A. Kadyk, and G. H. Trilling, Phys. Rev. Letters 7. 346 (1961). These authors give the branching ratio for $K^{+} \rightarrow \mu^{+}+\nu$ as $64 \%$, from which $\theta=0.269$. Also this value agrees with that from $K^{+} \rightarrow \pi^{0}+e^{+}+\nu$ within experimental errors.
}

${ }^{7}$ N. Cabibbo and R. Gatto, Nuovo Cimento $\underline{21}, 872$ (1961). Our notation for the currents is different from the one used in this reference and by Gell-Mann; the connection is $j_{\mu}{ }^{(0)}=j_{\mu}{ }^{1}+i j_{\mu}{ }^{2}, j_{\mu}{ }^{(1)}=j_{\mu}{ }^{4}+i j_{\mu}{ }^{5}$.

${ }^{8} \mathrm{~W}$. Willis et al. reported at the Washington meeting of the American Physical Society, 1963 [W. Willis et al., Bull. Am. Phys. Soc. 8,349 (1963) this branching ratio as $\left(0.9_{-0.5}^{+0.5}\right) \times 10^{-4}$. If it is allowed to vary between these limits, our predictions for the $\Sigma^{-} \rightarrow n e^{-} \bar{\nu}$ varies between $0.8 \times 10^{-3}$ and $4 \times 10^{-3}$, and that for $\Lambda^{0}$ $\rightarrow p e^{-} \bar{\nu}$ between $1.05 \times 10^{-3}$ and $0.56 \times 10^{-3}$. I am grateful to the members of this group for prepublication communication of their results.

${ }^{9}$ R. P. Ely, G。 Gidal, L。Oswald, W. Singleton, W. M. Powell, F。W.Bullock, G. E。 Kalmus, C. Henderson, and R。F。Stannard [Proceedings of the International Conference on High-Energy Nuclear Physics, Geneva, 1962 (CERN Scientific Information Service, Geneva, Switzerland, 1962), p. 445] give the branching ratio for $\Lambda \rightarrow p+e^{-}+\bar{\nu}$ as $(0.85 \pm 0.3)$ $\times 10^{-3}$, while that for $\Sigma^{-} \rightarrow n+e^{-}+\bar{\nu}$ is given (see preceding reference) as $(1.9 \pm 0.9) \times 10^{-3}$.

${ }^{10}$ R. P. Feynman, Proceedings of the Tenth Annual International Rochester Conference on High-Energy Physics, 1960 (Interscience Publishers, Inc., New York, 1960), p. 501. Recent measurements of the muon lifetime have slightly increased the discrepancy. We think that more information will be needed to decide whether our 3 rd assumption can be maintained.

\section{EXPERIMENTAL EVIDENCE ON $\pi-\pi$ SCATTERING NEAR THE $\rho$ AND $f^{\circ}$ RESONANCES, FROM $\pi^{-}+p \rightarrow \pi+\pi+$ NUCLEON, AT $3 \mathrm{BeV} / c^{\dagger}$}

V. Hagopian and W. Selove

University of Pennsylvania, Philadelphia, Pennsylvania

(Received 22 April 1963)

This note reports some preliminary results on $\pi-\pi$ scattering, near the $770-\mathrm{MeV} \rho$ and $1250-$ $\mathrm{MeV} f^{0}$ resonances. The experiment is the one reported earlier ${ }^{1}$; with more data measured (now about $75 \%$ of the two-prong events), we have examined the data to see to what extent they seem analyzable in terms of $\pi-\pi$ scattering. We give a brief summary of the results, and then a few details. A more detailed report will be available later.

(1) There is evidence of a major contribution from the one-pion-exchange mechanism ("peripheral collision"), for low nucleon recoil momentum. We take the region of $\Delta^{2}<\Delta_{\min }{ }^{2}+10$ to be interpretable in terms of $\pi-\pi$ scattering. $\left(\Delta^{2}\right.$ is the square of the four-momentum transfer to the nucleon, in units of the pion mass squared; $\Delta_{\text {min }}{ }^{2}$ is the lower kinematic limit, which is a function of the $\pi-\pi$ "mass" and the incident energy )
(2) We then consider these "peripheral" (i.e., peripheral-collision) events to be representative of the angular distribution of $\pi-\pi$ scattering. Two obvious points of caution must be mentioned here: (a) Interference effects arise from nucleon isobar production, and (b) the effective $\pi-\pi$ scattering is off the energy shell. From detailed examination of the data, we believe neither of these effects is so severe as to grossly affect the further conclusions below. A third possible complicating effect is interference from two-pion decay of the $\omega$, into $\pi^{+} \pi^{-}$; the possible magnitude of this effect is at present difficult for us to estimate.

(3) The spin of the $f^{0}$ is greater than zero, as reported earlier by Veillet et al. ${ }^{2}$ We believe it is difficult to draw any conclusion from these data as to whether the spin is 2 or greater than 2. (Isospin arguments, and the data directly, exclude spin 1.)

(4) The $\pi^{-}-\pi^{0}$ scattering in the $\rho$ region is con- 